LA-6961-MS

Informal Report
UC-20f

Issued: November 1977

\title{
Automated Computer Analysis of Plasma-Streak Traces from SCYLLAC
}

\author{
R. L. Whitman \\ F. C. Jahoda \\ R. P. Kruger
}


This work was supported by the Department of Energy, Division of Magnetic Fusion Energy.

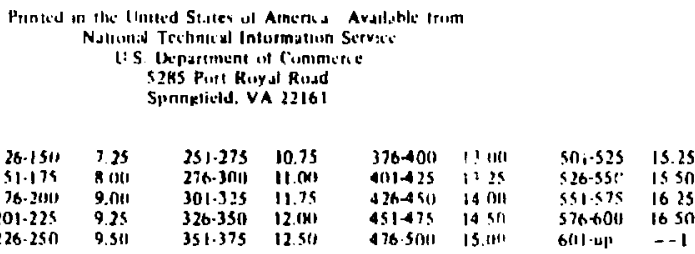

\begin{tabular}{|c|c|c|c|c|c|c|c|c|c|}
\hline Mlusutkhu & 53.1110 & 126.1511 & 3.25 & 251.275 & 10.75 & $376-400$ & $1 !$ & $5 n+525$ & $\begin{array}{l}\text { Is. } 25 \\
\text { is } 50\end{array}$ \\
\hline$\left(20 \cdot 35_{1}\right)$ & +40 & 176.300 & 9.04 & 301.325 & 1175 & $426-490$ & 14011 & $591 \leq 15$ & 1625 \\
\hline 1151.1175 & 325 & 201.225 & 9.2 & so & 12.01 & 451475 & 14501 & s7betod & $165 n$ \\
\hline 1176.1051 & 6.00 & $226-250$ & 9.511 & 351.375 & 12.50 & 476.5011 & is int & (II)-u| & --1 \\
\hline
\end{tabular}

I. Add s:.s11 fot cach additional 100.page increment from 601 papts up.

Thin rrport was pregased at an arciount of wipk spunuired be this krport was prepased al an arcount of wirk sbunwirrd

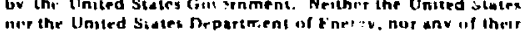

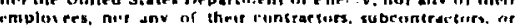
theit emplovers, makin an warrunty, enpress or implind, ot

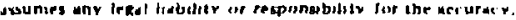

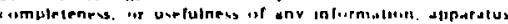

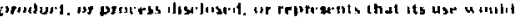
int infringe pristels ou neid rights. 


\title{
AUTOMATED COMPUTER ANALYSIS OF \\ PLASMA-STREAK TRACES FROM SCYLLAC
}

by
R. L. Whitman
F. C. Jahoda
R. P. Kruger

\begin{abstract}
An automated computer anaiysis technique that locates and references the approximate centroid of single- or dual-streak traces from the Los Alamos Scientific Laboratory SCYLLAC facility is described. The technique also determines the plasma-trace width over a limited self-adjustiıg region. The plasma traces are recorded with streak cameras on Polaroid film, then scanned aind digitized for processing. The analys is technique uses scene segmencation to separate the plasma trace from a reference fiducial trace. The technique employs two methods of peak detection; one for the plasma trace and one for the fiducial trace. The width is obtained using an edge-detection, or slope, method. Timing data are derived from the intensity modulation of the fiducial trace. To smooth (despike) the output graphs showing the plasma-trace centroid and width, a technique of "twicing" developed by Tukey was employed. In addition, an interactive sorting algorithm allows retrieval of the centroid, width, and fiducial data from any test shot plasma for post analysis. As yet, only a limited set of the plasma traces has been processed with this technique.

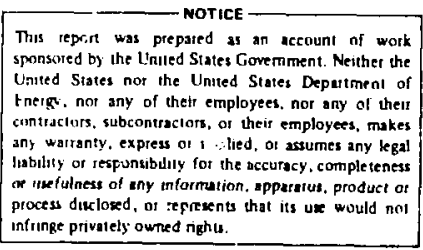

\section{INTRODUCTIDN}

At present, personnel in the LOS Alamos Scientific Laboratory (LASL) CTR Division manually trace the SCYLLAC plasma streak traces recorded on high-speed Polaroid film (Fig. 1). They need a high-speed, two-dimensional computer analysis technique to automate this tracing progress. We have developed such a technique for single- or dual-plasma streaks.*

*We will explain the processing method for an image with a single-plasma trace. The method is the same for dual-trace images except as noted in Secs. II and III. 
The Polaroid image in Fig. 1 contains a single-plasma trace and a fiducial trace. The fiducial trace allows the unfolding of magnetic field interference generated by the SCYLLAC while the plasma trace is being recorded. The twodimensional computer analys is technique segments the digitized image into two parts, the first contains the plasma trace from initial implosion to the end of the image and the second contains the fiducial trace over the same time interval. These parts are labeled $A$ and $B$ in Fig. 1. Following segmentation, the scan 1 ines of the plasma trace are smoothed. The resultant image is processed by a peak-detection algorithm designed to follow the approximate centroid (position of maximum intensity) over a limited self-adjusting region. The region extends from the initial implosion (Point 1) to a point where the trace merges with the backgruund noise (Point 2). Each scan line in the segment containing the fiducial trace is then processed to detect the peak over the same interval. The period (in microseconds) of the modulated intensity of the fiducial trace is also determined. The plasma trace width is determined using the initial coordinates of the plasma centroid and the edge-detection, or slope, method. ${ }^{1}$ The information used in edge detection is usually valid over a limited region of the plasma trace because of boundary considerations. The boundary constraints can be seen by the width information shown in Fig. 2, relative to Fig. 1 .

The referenced plasma centroid edges, and plasma-trace width are then smoothed using a nonlinear filter technique developed by Tukey, ${ }^{2}$ called "twicing." The original referenced data and the filtered data are shown in Figs. 3 and 4 , respectively.

The final step in handling the processed data is to generate individual files that contain label information. Labeling, based on the user's requirements, is used in conjunction with a sorting routine that can locate a file containing a particular test or other descriptors. This sorting capability allows the user to further analyze. the extracted data.

Although only $16 \mathrm{plasma}$ streak traces have been processed to data, all have been successful in that the centroid, width, and timing parameter are determined.

\section{FROCESSING METHOD}

A. Simple Scene Segmentation

When automating the analysis of the plasma trace, the first step is to decompose the image (scene) into its principal spatial components. This limits the specific processing to thosa regions that contain the plasma trace and 
fiducial trace. The segmentation can be partially accomplished when the traces are scanned. However, a more region-specific segmentation of the image into plasma and fiducial trace regions is also needed. This is determined by average vertical profiles of the plasma trace and fiducial trace, like the one in Fig. 5. Because the images are digitized perpendicular to the arientation of the traces: each vertical profile point is the average of the corresponding digitized points or pixels in each scan line. After obtaining the average profile, its first derivative is calculated by taking the derivative of a second-degree least squares polynomial fit through the four nearest intensity values on either side of the given center point (Fig. S). (Refer to the appendix for a more detailed description.)

These derivative values are then searched for the two largest positive and negative values. The first such values encountered are shown as Points 1 and 2 , respectively, in Fig. 6. They represent the positions of largest slope on the bottom and top sides of the fiducial trace (Points 1 and 2 in Fig. 5). In the same search, no other large positive derivative is found but the second large negative derivative is encountered at Point 3 in Fig. 6 . This point represents the top side of the plasma trace (Point 3 in Fig. 5). Points 4 and 6 are obtained by searching in the appropriate direction from Points 1 and 3 until a predetermined minimum threshold is reached (Fig. 5). Point 5 is determined by searching in the appropriate direction from Point 2 until ninimum is reached within a prerstermined distance. Points 4,5 , and 6 are used in the first segmentation to separate the plasma and fiducial-trace regions and to remove a major portion of the scanned background from subsequent processing.

After the initial scene segmentation, the average horizontal profile is calculated for the plasma trace only (see Fig. 7). Because the traces are digitized perpendicular to the time, or horizontal, axis each horizontal profile point is the average value of the pixels from Puints 4 to 6 in each scan line. inen the first derivative of this profile (Fig. 8) is computed, using the same technique as that used for the vertical profile.

The derivative values are searched for the largest positive value (Point 1 in Fig. 8) which represents the steepest slope of the initial implosion area (Point $l$ in Fig. ?). Point $l$ is used in the final segmentation to separate the: plasma trace from the remainder of the background. All further processing of the plasma- and fiducial-trace segments begins at Point 1 on the time axis. 


\section{B. Presmoothing and Detection of the Plasma Centroid}

We now apply one-dimensional smoothing to the plasma trace along the scan lines. This presmoothing reduces the digitized film and scanner noise, thus dec.easing the probability of a false lock on the plasma-trace centroid or line of maximum intensity. We are assuming that the plasma has an approximate Gaussian shape when projected onto the two-dimensional image plane.

The presmoothing is performed by a 15-point least squares parabolic fit through the 7 nearest intensity values on either side of the given point. However, seven points are lost at the beginning and end of each smoothed line. By repeating the first smoothed point for the first seven points and the last smoothed point for the last seven points, the smoothed image remains the same size as the unsmoothed image, which simplifies subsequent processing.

The next processing step is to detect the position of the plasma-trace centroid. Figure 9(a) shows a typical cross section (smoothed line) perpendicular to the plasma trace's horizontal axis (Fig. 2). The slope at any given point along the cross section is calculated as the derivative of a second-degree least squares polynomial fit through the nearest intensity value on each side of the given point [Fig. $9(b)]$. The first and last derivative values are then repeated once to make the derivative line the same number of pixels as the original line. This procedure is repeated for all the lines in the plasma-trace segment.

Based on the first derivative of each line of the smoothed plasma-trace segment, a search is started to find the one or two largest values per line. The user specifies the number of plasma traces in the segment which determines whether one or two maxima are sought.

For any point to be considered as a maximum, the derivative about the point must have at least five consecutive positive first-derivative points followed by at least five negative derivative points. These conditions imply processing only in the direction in which the derivative was calculated. They also put a constraint on how close the maxima can be to one another and how well-defined they must be. These maximum points must also lie above the average value of their line of smoothed data. For the maxima in lines other than the first line, a window of plus and minus 10 points irom the position of the maximum value in the previous line is calculated. If the user thi..ks the amount of noise in the plasma-trace segment is high enough, an amplitude window, based on the previous maxinum plus and minus $50 \%$ of the standard deviation of the present line data, can be employed. 
If no maximum is found within the position window, that constraint is lifted and a second search is initiated. On the second search, if no maximum is found to meet the amplitude window, or if the maximum found has moved a significant distance from where it was in the previous line, the centroid-following technique is terminated. When two traces are present, they may join and later bifurcate but they may not cross without giving an erroneous result. Also, when they merge for more than $50 \%$ of the remaining plasma segment, the trace taking the largest jump to merge is terminated at that point, and only one trace is followed thereafter.

C. Peak Detection of the Fiducial Trace

The detection of the maximum of ine fiducial-trace segment is simple compared to that of the plasma trace, because the signal-to-noise ratio (SNR) is very high in the fiducial-trace segment. Thus, the peak-detector algorithm can be simpler. The peak detection begins at the same line (Point 1 in Fig. 7) as the plasma trace and ends at the same line at which the plasma trace is lost. The only modification to tine presmoothing algorithm is that a moving window seven points in width is placed about: each new maximum, as it is found, to narrow the area of search for the next maximum. This operation is necessary because the fiducial trace passes very close to the initial confinement, or implosion area, of the plasma trace. When the segmentation is performed, part of this initial confinement area is olaced within the fiducial segment. Because the intensities of the fiducial trace and plasma-trace confinement area are of conparable amplitude, a false lcck could result without the addition of the windowing step. The result of this technique applied to the fiducial trace in Fig. 1 is shown in the fiducial-trace position plot in Fig. 10. The initia? positive slope in the $X-Y$ position is due to the magnetic interference by the SCYLLAC on the recording streak camera. The fiducial peak-detection step defines the fiducial position needed to unfold this interference from the plasma trace.

D. Timing Information from the Intensity-Modulated Fiducial Trace

The fiducial trace is intensity modulated by an R-C network. The period of the modulation is needed to verify the timing settings used in recording that particular plasma trace.

When the modulated fiducial trace is recorded on the Polaroid film and later digitized, the modulation waveform is basically triangular. This is due to the effect on the modulated ivaveform by the logarithmic characteristics of the film. To calculate in microseconds the period of the waveform, a one-dimensional 
Fast Fourier Transform (FFT) is obtained of the peak intensity values of the fiducial trace. The position (N) of the largest harmonic other than the dc harmonic is extracted from the FFT calculation. It agrees with the number of samples (points) per centimeter (SPC) obtained from a digitized Polaroid image containing a centimeter grid at the same magnification as that of the plasma streak trace. We can then calculate the number of cycles per centimeter, or frequency, of the largest harmonic (FLH). Given the number of microseconds per centimeter (MPC), we can calculate the period of the largest harmonic,

$$
\text { PLH }=\text { MPC/FLH (microseconds). }
$$

The values of PLH and SPC are incorporated in the final output file sets that describe each plasma streak trace or traces.

E. Edge Detection for Determining the Plasma-Trace Width

After obtaining the position of the centroid of the plasma trace by use of the first derivative and window constraints, a gradient maximization method is used to determine the edges of the plasma trace. The detached edges are specified as those puints along each vertical scan line that are closest to the previously calculated plasma centroid and have the largest local gradient. These points correspond to the maximum (Point 1) and minimum (Point 2) first-derivative values closesi to the centroid, as shown in Fig. 9(b).

This process is repeated for all vertical scans for which a plasma centroid was found. No detected edge value is retained for those maximum gradient locations which violate boundary conditions established when the presmoothing and derivative images were computed. Instead, the edge position is filled with a zero for $X$ and $Y$ values. Once the position of the edge has been acquired, the previous points in the plasma segment outside the edge points are zero filled (upper and lower edges are done independently). The resulting edges for the single-plasma trace in Fig. 1 are shown in Figs. 2 and 3 . Note that the maximumslope method for edge detection places the plasma-trace edge at approximately the same place a human observer would visually select.

The width of the plasma trace is now calculated by subtracting the lower edge position from the upper edge position over the interval where both are nonzero (Fig. 11). 
F. Referencing of Plasma Centroid and Edges

The magnetic interference is now removed from the plasma centroid and upper and lower edges by subtracting line by line the vertical position of the fiducial trace from the plasma-trace positions. The plasma width does not need correcting, because each edge of the plasma trace is equally affected. The resultant referenced curve is shown in Fig. 3.

G. Smoothing by Twicing

To smooth and despike the plasma trace centroid, edges, and width, a technique of "twicing" developed by Tukey ${ }^{2}$ was employed. This nonlinear technique compensates for two limitations of linear smoothing. These are

- the errors in the resulting smoothed data caused by large single-element or short-burst noise spikes, and

- loss of valid high-frequency information.

The smoothing technique, using a moving window as input, calculates a value as output. This deals with the first limitation. Twicing deals with the second limitation. The twicing process is as follows. Once the original data have been filtered with a median filter of nine points, the median-filtered data are subtracted from the original data to obtain a residual. Next, a median filter is applied to the residual and is added to the original median-filtered data. The original data and the twiced data can be seen in Figs. 3, 11, 4, and 12 . H. Generation of Files Containing Original and Smoothed Data and Retrieval for Postprocessing

When postprocessing is desired, it is necessary to internally identify and record each set of original and smoothed data for a particular shot on an individual file. To do this, we use a small subset of the Los Alamos Digital Image Enhancement Software (LADIES) ${ }^{3}$ library previously used in the initial data processing.

The internal identification code attached to each file is four words. These are a subset of the 10 words that are alterable by a LADIES library program called SET. These four words may contain any display code information. The following is the identifying code in each word used to differentiate the content of each file. Note that there are 10 alphanumeric display characters per word.

IDENTIFICATION CODE

WORD $1=X X X X X B C C B$ RR

$X X X X$ - SCYLLAC Shot Number

0 to 9999 
CC - Camera Number

0 to 99

$R R$ - Rack Nurnber

0 to 99

B - Blank

WORD $2=1$ - Single-plasma trace

2 - Dual--plasma trace

WORD $3=1$ - Referenced centroid of an upper plasma trace of a dual trace or referenced single-plasma trace (Refer to Word 2)

$=2$ - Referenced lower piasma-trace centroid

$=3$. Fiducial trace

$=4$ - Width of upper piasma trace

$=5$ - Referenced upper edge slope $(-)$ of the upper plasma trace or single trace

$=6$ - Referenced lower edge slope $(+)$ of the upper plasma trace or single trace

$=7$ - Width of lower plasma trace

$=8-$ Referenced upper edge slope $(-)$ of the lower plasma trä..c

$:=9$ - Referenced lower edge slope $(+)$ of the lower plasma trace

WORD $4=1$ - Referenced original data

$=2$ - Referenced twice-Tukey-filtered data

$=3$ - Period and amplitude data

With the four words altered by SET, the processed data plus descriptor words are written to an individual file. Thus, a disk file can be assigned containing many files of the processed SCYLLAC data. Each of these files can be searched and postprocessed.

An additional sorting program was written to input the encoded data files. The program concept was based on a priori user knowledge of which shot data were desired. Through an interactive question-and-answer session, the user could describe the needed data by use of the identification code. The program wolild then retrieve the four identification words from each file, and compare them with the code requested. When the file is found, it is set aside in an array 
immediately available for postprocessing. This sorting continues until all the needed files are retrieved and the user requests to exit from the program.

\section{TWO EXAMPLES OF RESULTS}

The last section discusses the various components of the algorithm necessary to extract the referenced centroid and width. Two examples wiil be presented, illustrating the processed results on a single- and dual-plasma trace.

For the familiar single-plasma trace case in Fig. 1, the corresponding processed trace is shown in Fig. 2. The bolder white center overlay plot shows the position of the centroid. The fainter white upper and lower overlay plots represent the position of the edges. Figure 3 is the plot of the referenced centroid and width abstracted from Fig. 2. From the raw data used in the edge plots in Fig. 3, a limited plot of the width of the plasma is found as shown in

Fig. 12. The raw data in Figs. 3 and 11 is then twice Tukey filtered, resulting in Figs. 4 and 12.

The dual-trace examples are shown in Figs. 13 and 14. The bolder white center overlay plots outline the approximate position of the centroids. The less apparent white upper and lower overlay plots represent the position of the edges of each plasma trace. Figures 15 and 16 display the referenced centroids and edges extracted from Fig. 14. The width of each trace is calculated over a limited interval (Figs. 17 and 18). The original daia plots in Figs. 15-18 are twice Tukey filtered, and the results are shown in Figs. 19-22, respectively.

In Figs. 14 and 15, note that the upper trace data are not processed correctly from Point 1 (almost ihe beginning) to Point 3 because of the initial faintness of the upper trace and the closeness of the upper and lower traces at Point 2. Also note that all the lower trace data are correct to Point 4 . The upper trace data are good from Point 3 to Point 4. The traces join at Point 4, and the data beyond that point are not used.

The user must initially specify whether a single- or dual-plasma trace is present. He may change several other parameters, such as shot number, rack number, camera number, and number of traces to be processed, but they do not affect the processing. Fifteen additional parameters may be changed to fine tune the process, however this is usually unnecessary. 


\section{CONCLUSIONS}

An automated computer analysis technique for processing single- and dualplasma streak traces has been developed. The technique has been applied to 16 plasma streak traces, both single and dual. The examples in Secs. II and III show that the lechnique perfornis better on single traces than on dual traces.

The processing time on a CDC 7600 for an original $512 \times 512$, 6-bit graylevel image is nearly indeperident of the number of traces on the image. Either a single- or dual-trace image requires $30 \mathrm{~s}$ or less.

Both the automated processing technique and postprocessing sorting algorithm are available on the CROS and LTSS systems at LASL, as well as on the CTR-LTSS system at Lawrence Livermore Laboratory (LLL). This will allow for a high degree of flexibility and through-put potential in the processing of the SCYLLAC Plasma streak traces.

\section{REFERENCES}

1. R. H. Selzer, D. H. Blankenhorn, D. W. Crawford, S. H. Brooks and R. Barndt, Jr., "Coinputer Analys is of Cardiovascular Imagery," Proc. of Cal. Tech/JPL Conf. on Image Proc. Technology, Data Sources, and Software for Commercial and Scientific Applications, November 3-5, 1976, pp. 6-1, 6-20.

2. John W. Tukey, "Robust/Resistant Smoothing," Selected Examples of ERDA Research in the Mathematical and Computer Sciences, ERDA-76-118, pp. 80-82, September 1976.

3. B. R. Hunt and D. H. Janrey, "Digital Image Processing at Los Alamos Scientific Laboratory," COMPUTER Magazine, pp. 57-61, May 1974. 


\section{APPENDIX}

DERIVATIVE OF A SECOND-D「GREE LEAST SQUARES POLYNOMIAL

If $Y_{1}, Y_{2}, Y_{3}, \ldots$ are the intensity values along the scan line, $D_{n}$ is the derivative at the $n$th point of the polynomial through the nearest $\pm K$ points, then

$$
D_{n}=C_{K} \sum_{I=-K}^{K} I Y_{n+I} \text {, }
$$

where $c_{K}=12.0 / K\left(k^{2}-1\right)$. 


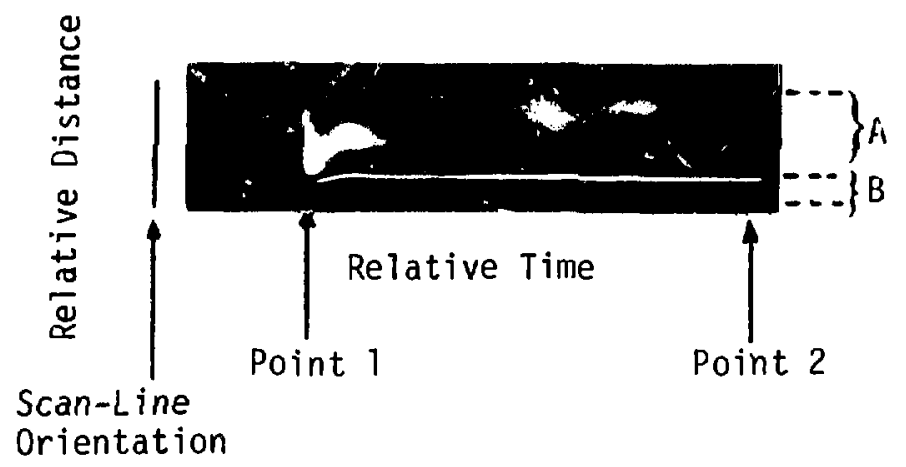

Fig. 1. Original image of a single-plasma streak trace $(A)$ and referencing fiducial trace $(B)$.

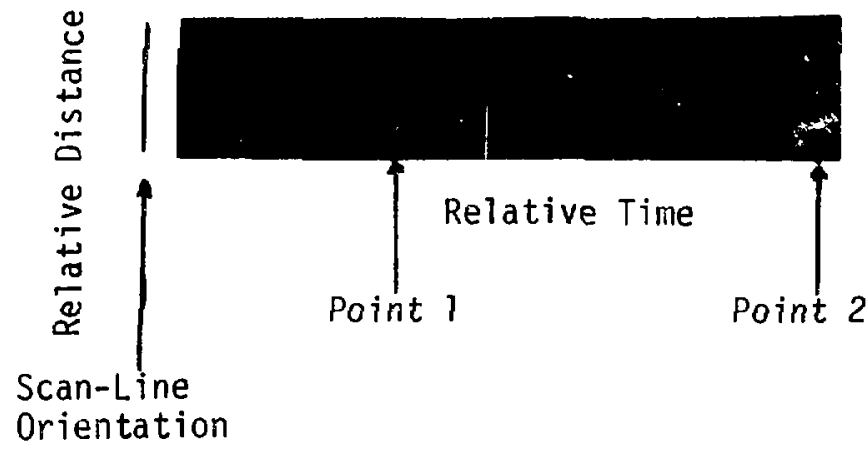

Fig. 2. The plasma trace in Fig. 1 with the centroid and edges outlined. 


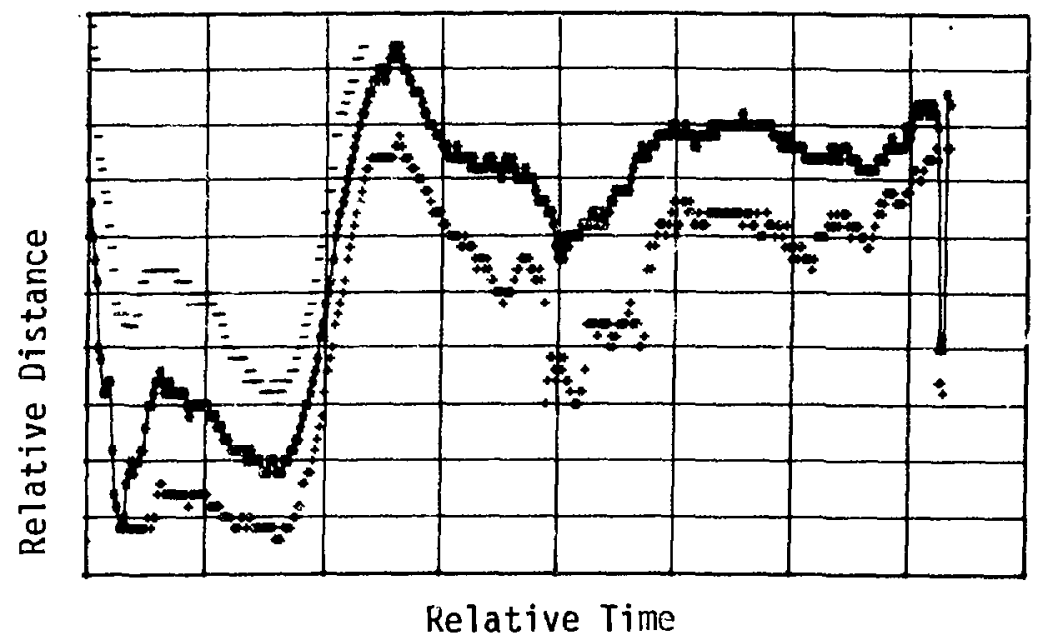

Fig. 3. Referenced centroid $(*)$ and edges $(-,+)$, original data.

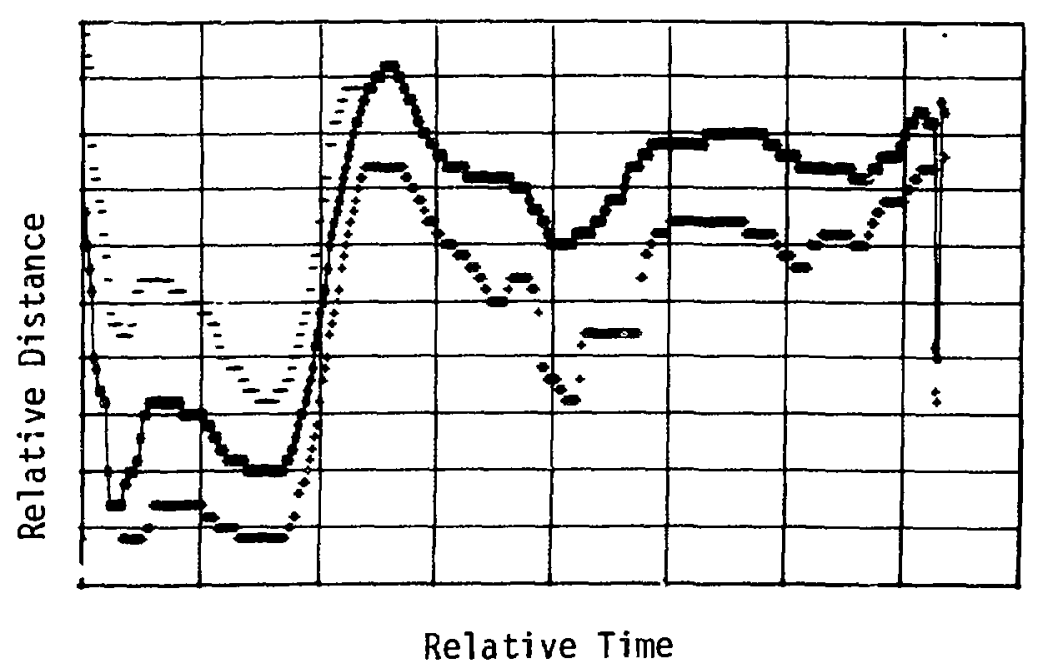

Fig. 4. Referenced centroid $(*)$ and edges $(-,+)$, filtered data. 


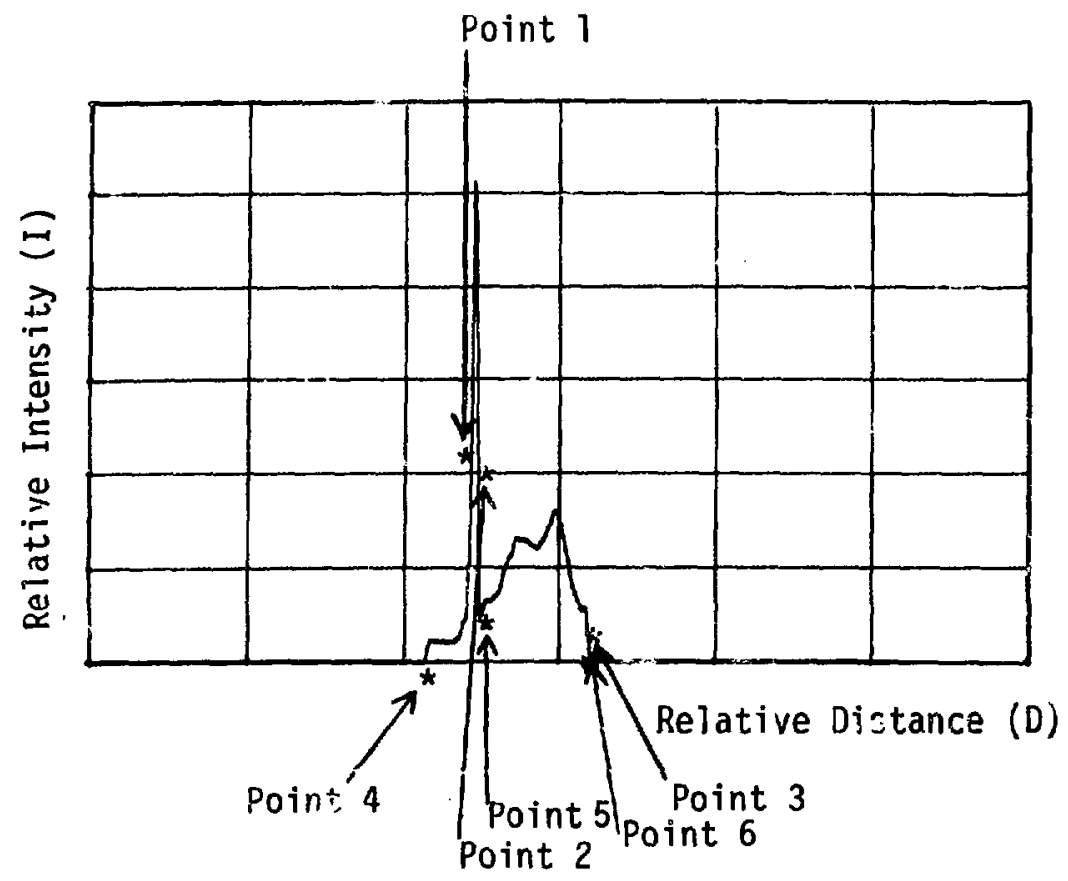

Fig. 5. Average vertical profile of Fig. 1.

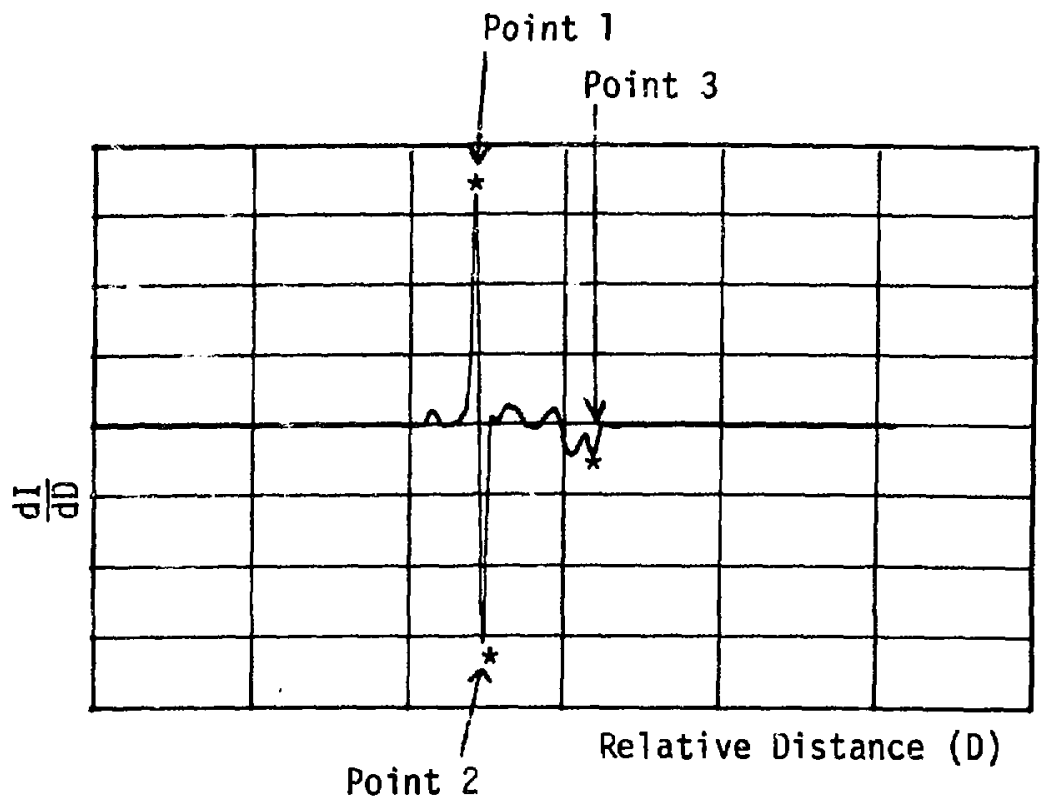

Fig. 6. First derivative of the vertical profile in Fig. 5. 


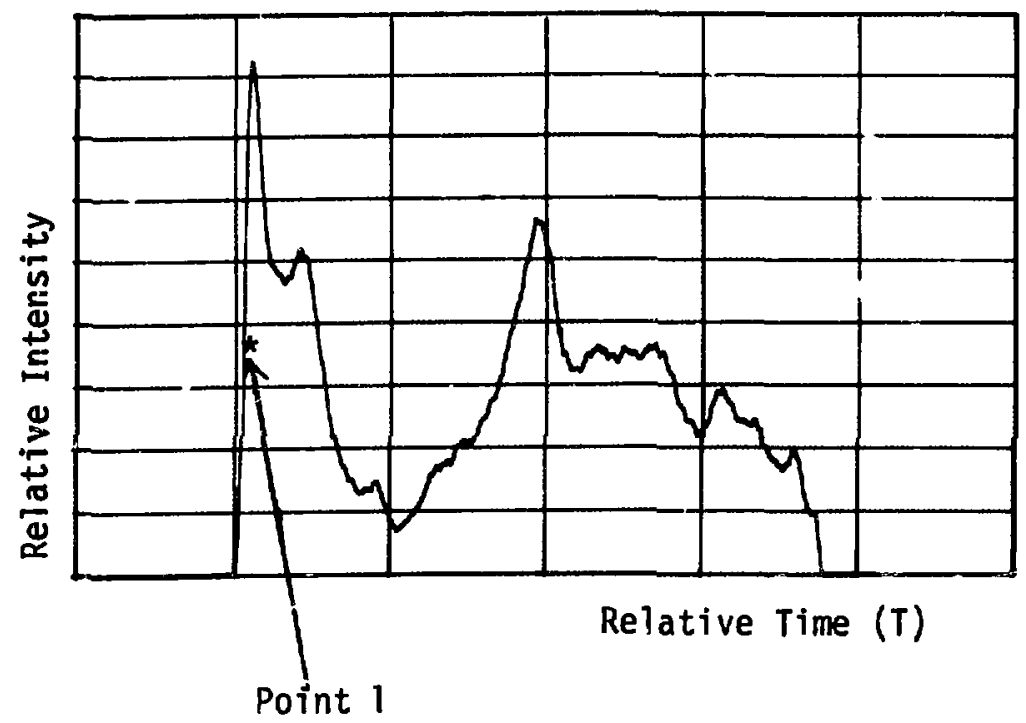

Fig. 7. Average horizontal profile of Fig. 1.

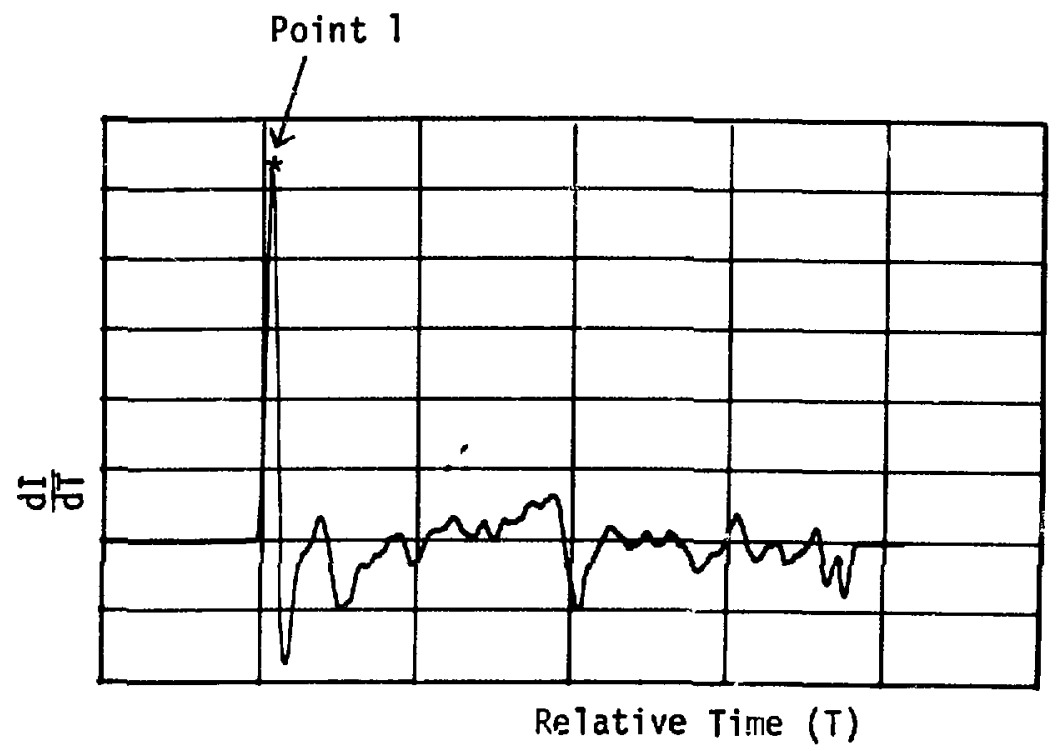

Fig. 8. First derivative of the average horizontal profile in Fig. 7. 


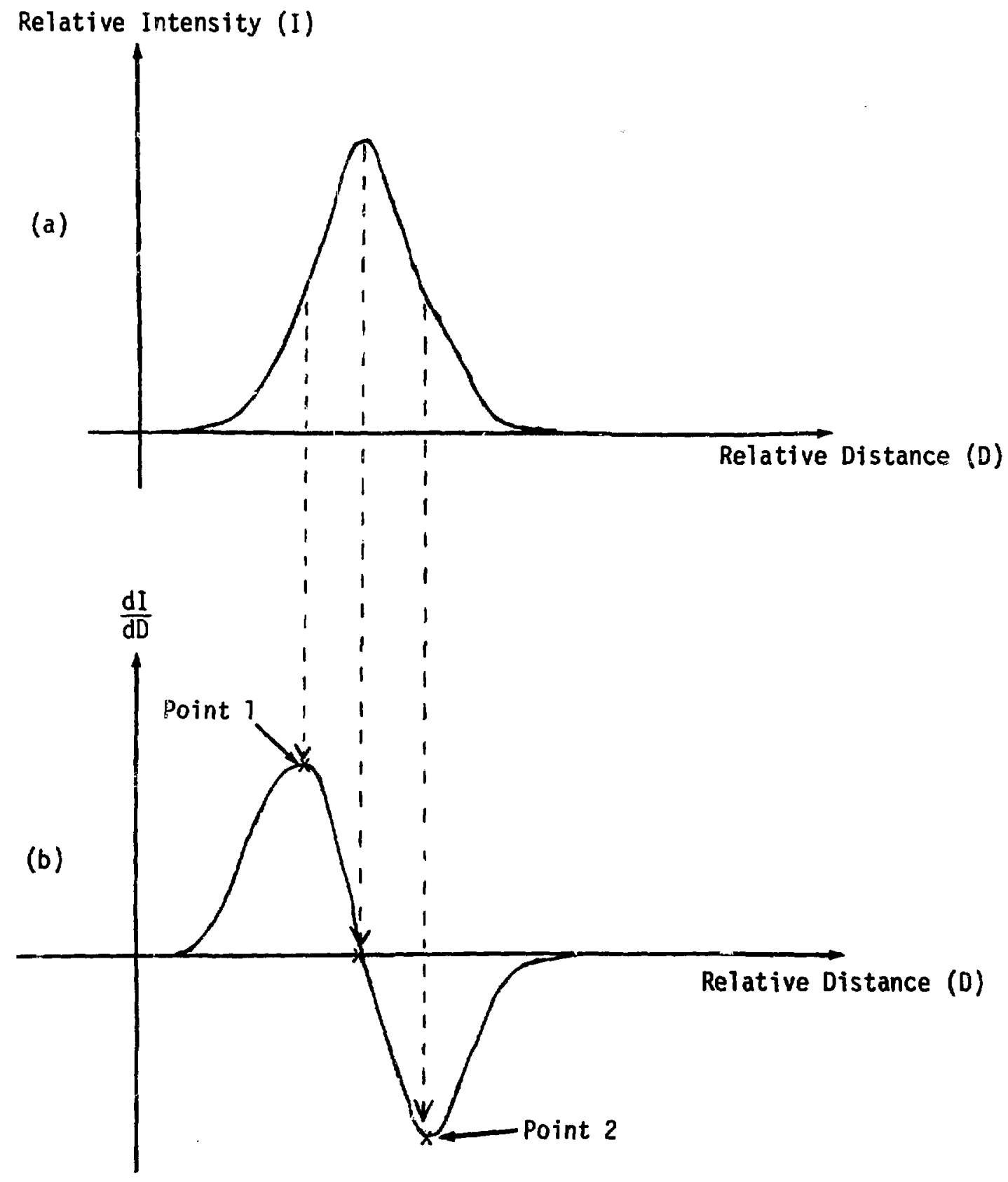

Fig. $9(a)$. Cross section through the plasma trace in Fig. 2. 9 (b). First derivative of the cross section in (a). 


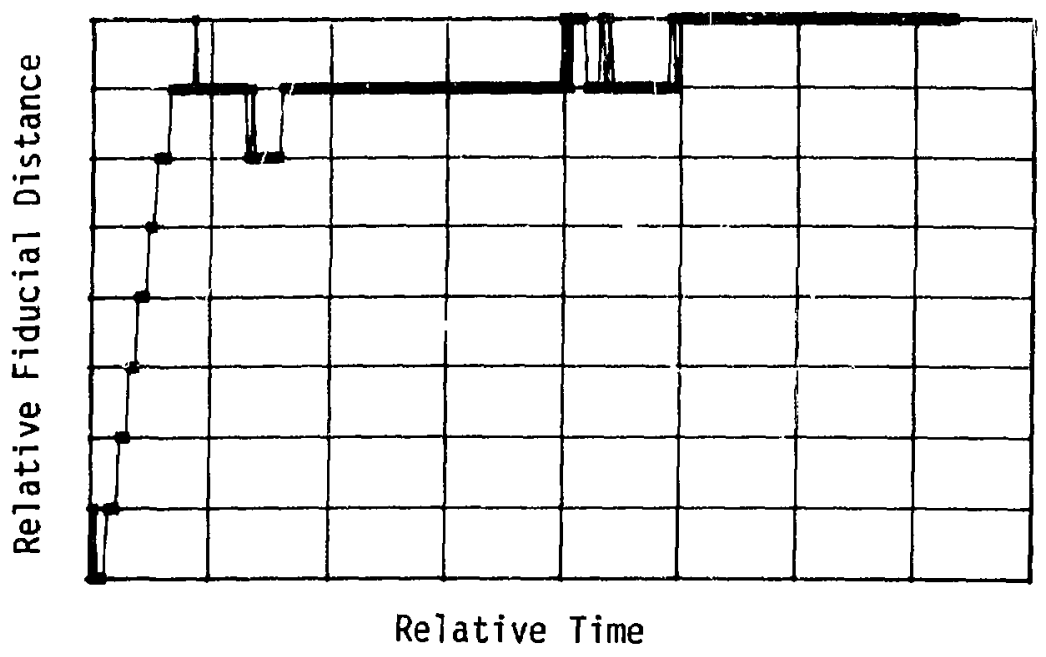

Fig. 10. Fiducial-trace position, raw data.

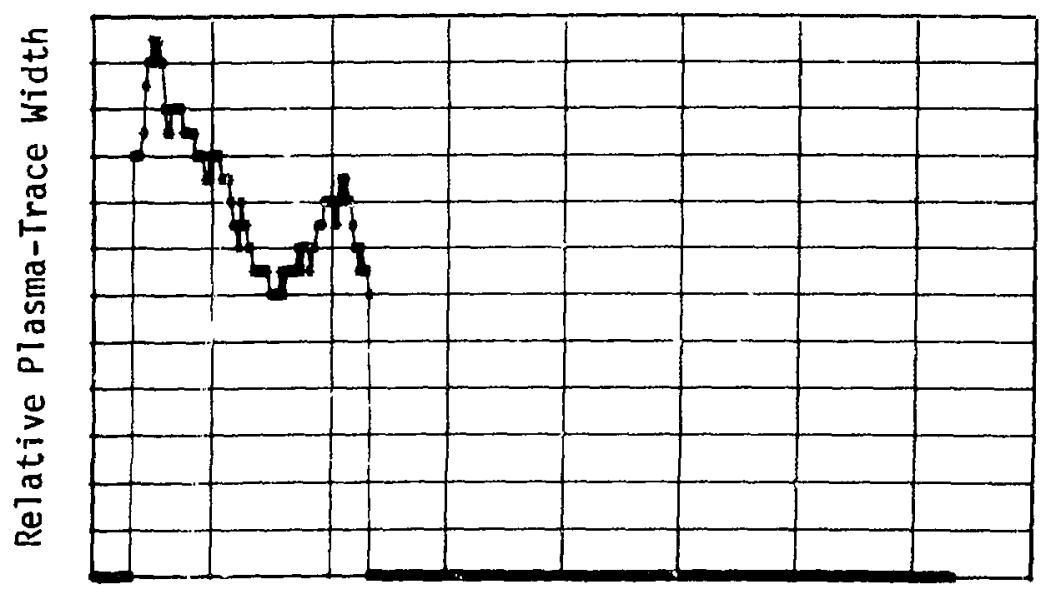

Relative Time

Fig. 11. Plasma-trace width, original data. 


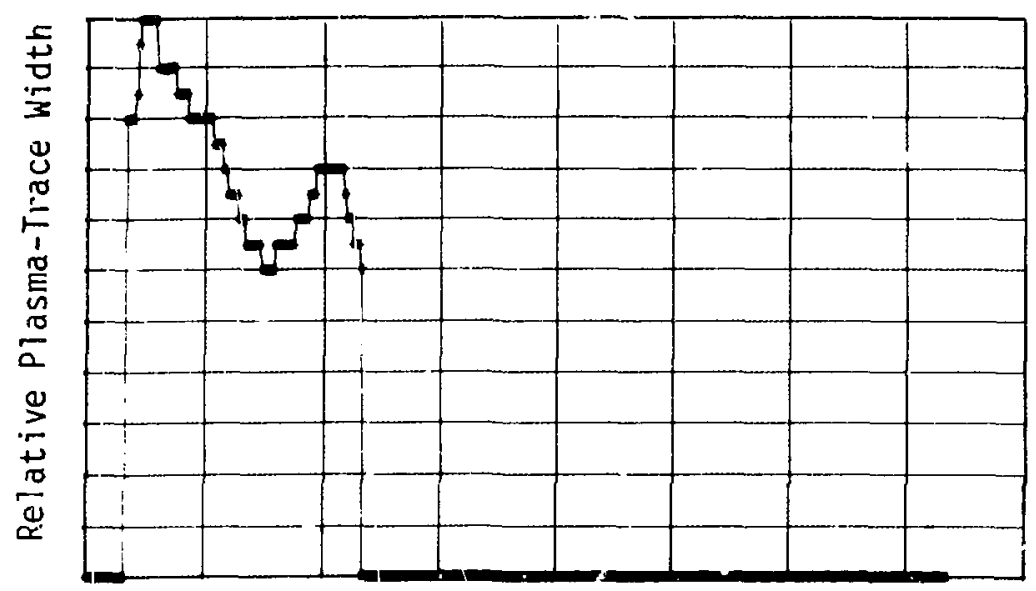

Relative Time

Fig. 12. Plasma-trace width, filtered data.

a

Scan-Line

Orientation

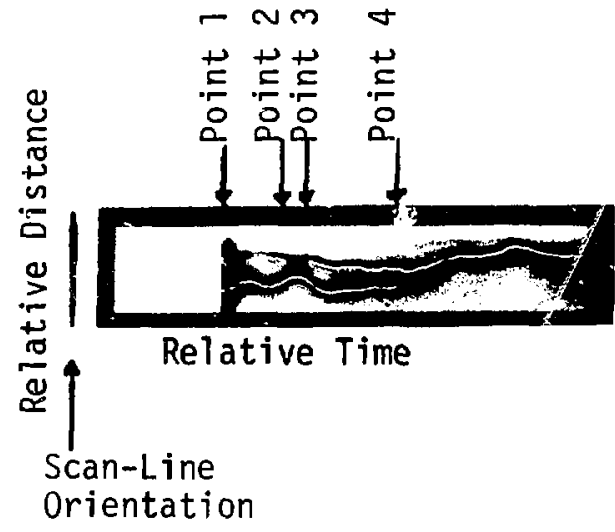

Fig. 14. The plasma traces $i$ -

Fig. 13 with the centruids and edges outlined. 


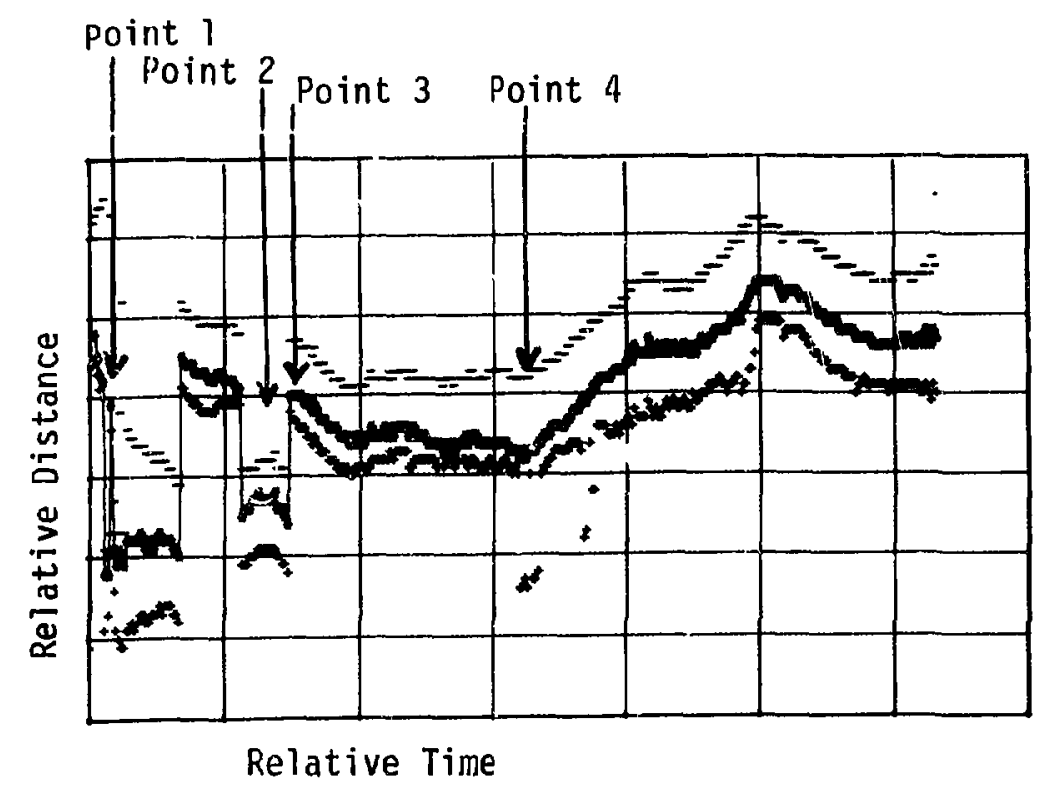

Fig. 15. Upper trace, referenced centroid $(*)$ and edges $(-,+)$, original data.

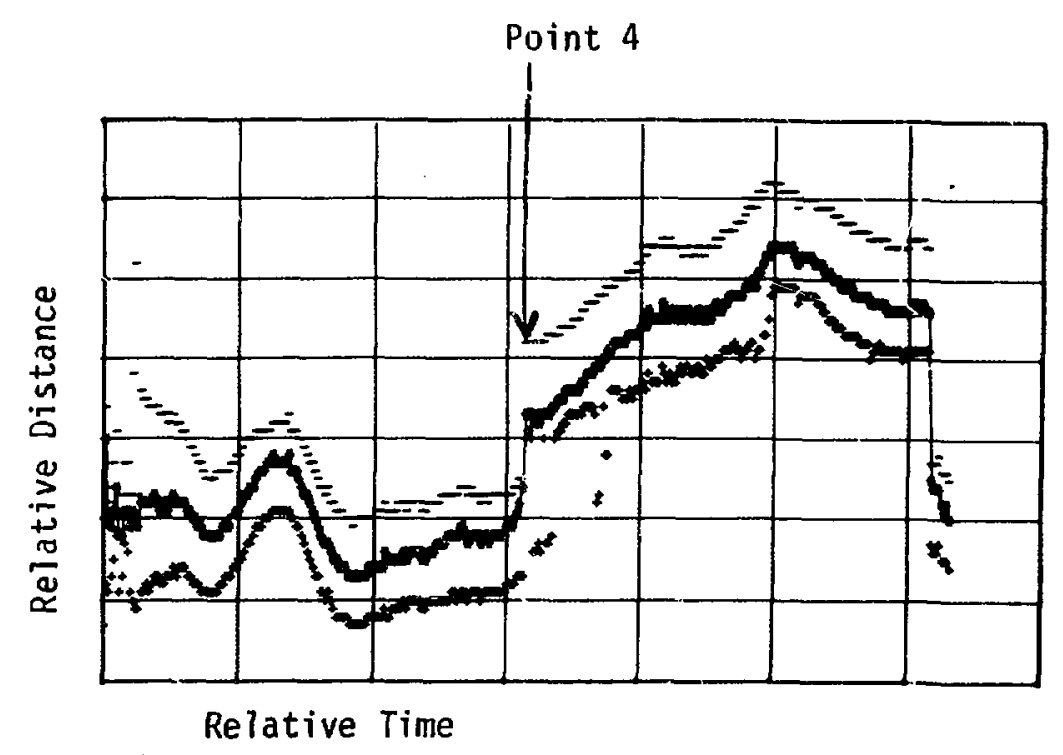

Fig. 16. Lower trace, referenced centroid (*) and edges $(-,+)$, original data. 

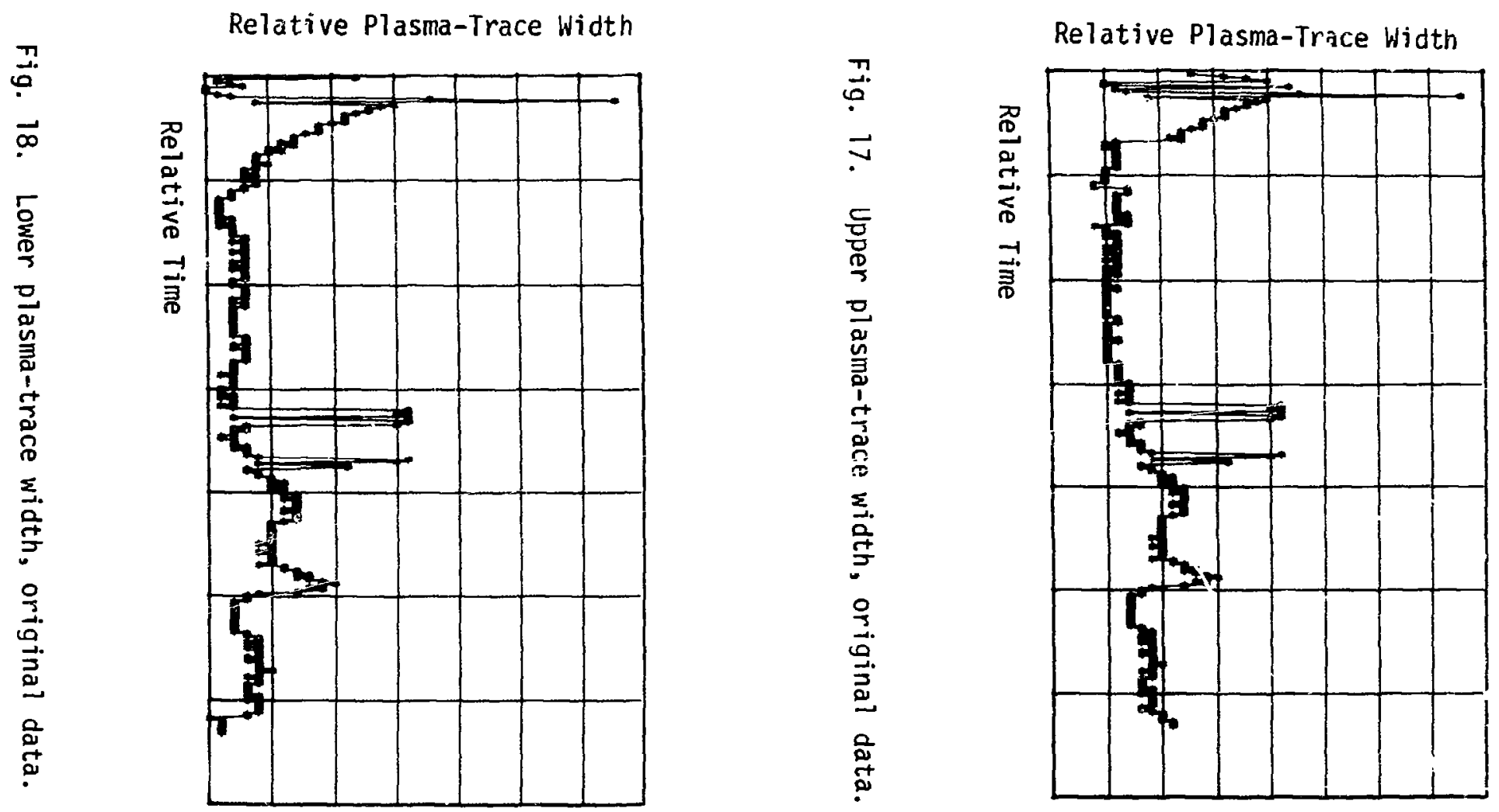
Relative Distance

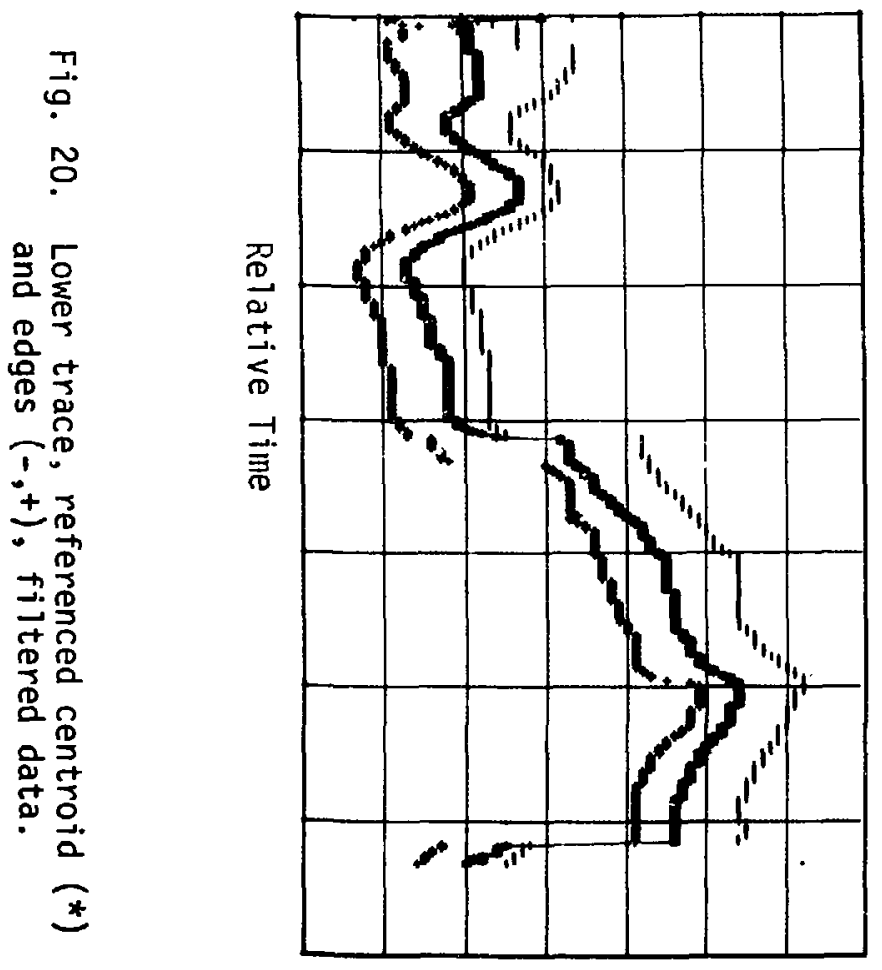

Relative Distance

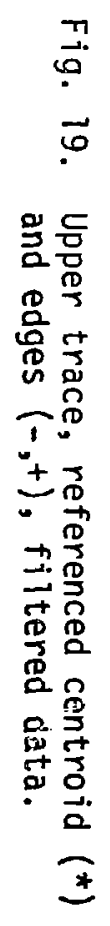




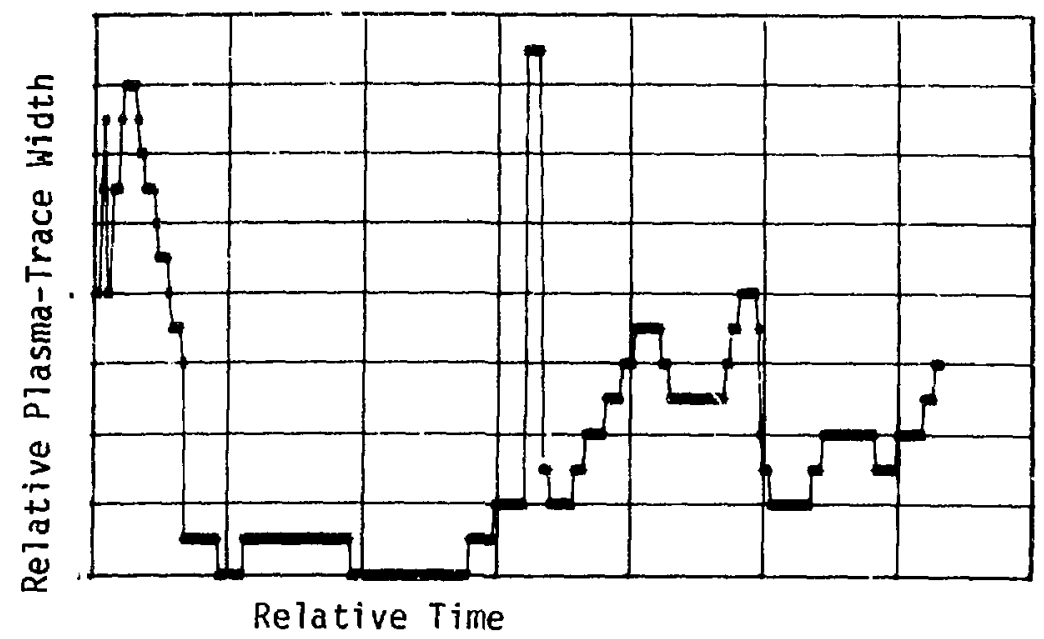

Fig. 21. Upper plasma-trace width, filtered data.

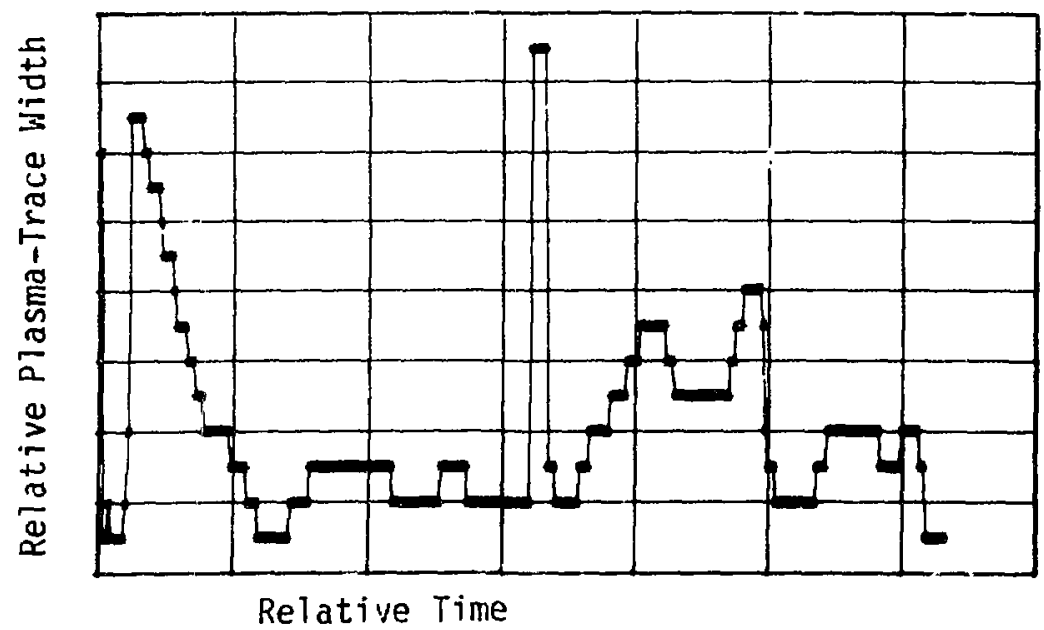

Fig. 22. Lower plasma-trace width, filtered data. 